\title{
Pulmonary embolectomy using normothermic venous inflow occlusion
}

\author{
D. B . C L A R K E \\ From Queen Elizabeth Hospital, Birmingham
}

Five patients are presented who underwent emergency pulmonary embolectomy with the use of venous inflow occlusion at normothermia. Two of these patients made satisfactory recoveries. It is suggested that this technique is of value in centres where facilities for providing cardiopulmonary bypass are not available.

The introduction of cardiopulmonary bypass for the urgent removal of massive pulmonary emboli by Sharp (1962) revolutionized the surgical management of this emergency. More patients were successfully treated in the four years following this than in the preceding 50 years since Trendelenburg's (1908) classical description of pulmonary embolectomy. The use of an extracorporeal circulation, and accurate diagnostic methods such as pulmonary angiography and radioactive isotope scanning of the lungs, must be regarded as the ideal. This ideal is difficult to attain in hospitals without the services of staff experienced in open-heart surgery and where facilities to provide a heart-lung machine, primed and ready to use within a very short time, do not exist.

There have been many recorded instances of successful pulmonary embolectomy without cardiopulmonary bypass, notably by Allison, Dunnill, and Marshall (1960), who used moderate hypothermia, and by the classical Trendelenburg technique (Kirschner, 1924 ; Steenburg, Warren, Wilson, and Rudolf, 1958). Vossschulte, Stiller, and Eisenreich (1965) were successful in four out of seven embolectomies using the technique of venous inflow occlusion at normothermia.

The following case reports are presented in support of the belief that simple methods of removing massive pulmonary emboli still have a place in circumstances of extreme urgency where more sophisticated techniques are not available.

\section{THE OPERATION}

Heparin which may have been given is neutralized by the appropriate dose of protamine. During the induction of anaesthesia there may be alarming hypotension. Vasopressor agents may need to be used.

The heart is exposed through a median sternotomy. Nylon tapes are passed round the superior and inferior venae cavae. These vessels may be occluded by either a rubber tube tourniquet or the application of vascular clamps.

A side clamp is applied to the pulmonary artery in its long axis and the portion of arterial wall included in the clamp is incised for $2 \mathrm{~cm}$. Four stay sutures are inserted.

At normothermia the circulation can be safely interrupted for three minutes. The superior and inferior venae cavae are occluded and the heart is allowed to empty. A timekeeper starts a stopwatch as soon as the carotid pulse is no longer palpable. The clamp is removed from the pulmonary artery and the small quantity of blood which escapes is removed with a sucker.

A right-angled Dejardin common duct forcep is a satisfactory instrument for exploring the pulmonary artery. Its curvature allows it to be passed into the lower lobe artery on either side. Clots can be grasped and extracted and small fragments can be flushed out by means of a Canny Ryle syringe filled with saline and a catheter, and by suction. After three minutes the lips of the incision in the pulmonary artery are lifted up by the stay sutures, the side clamp is reapplied, and the caval tourniquets are released. The heart usually takes over the circulation without difficulty and an improvement in haemodynamics is immediately apparent. If it is felt that exploration has been incomplete the procedure can be repeated after an interval of 15 minutes. Three minutes usually allows ample time for probing both sides 
of the pulmonary vascular tree. The incision in the pulmonary artery is then closed with an atraumatic suture. A period of observation is advisable before the chest is closed, and this time can be well used in narrowing the inferior vena cava in order to trap further emboli. It has been our practice to expose the inferior vena cava in the abdomen and to narrow it to a diameter of $4 \mathrm{~mm}$. by a stout ligature. Anticoagulation therapy is commenced as soon as the mediastinal drainage tubes have been removed.

\section{CASE REPORTS}

CASE 1 A man of 35, who was being investigated for a right pleural effusion, suddenly developed a feeling of constriction in the chest associated with a desire to defaecate. On examination he was pale and sweating with a systemic blood pressure of $100 / 60$ $\mathrm{mm}$. Hg. The right ventricular impulse was marked, the pulmonary component of the second heart sound was accentuated, and an apical third heart sound was present. The chest radiograph and electrocardiogram showed changes consistent with a diagnosis of pulmonary embolism. There were no signs of venous thrombosis in the calves. He was given heparin by intravenous injection. His condition deteriorated during the next 12 hours, and the blood pressure fell to $80 / 60 \mathrm{~mm}$. Hg. Pulmonary embolectomy was performed 13 hours after the onset of symptoms, and the pulmonary artery was explored using venous inflow occlusion at normothermia for two periods of 3 minutes 25 seconds and 2 minutes 40 seconds. Two emboli, $1 \mathrm{~cm}$. in diameter, and a number of smaller clots were removed (Fig. 1). The systolic pressure rose to $140 \mathrm{~mm}$. $\mathrm{Hg}$ when the circulation was re-established. The inferior vena cava was narrowed to $4 \mathrm{~mm}$. diameter with an encircling ligature.

His post-operative course was uneventful. Anticoagulant therapy with phenindione was begun 24 hours after surgery. The electrocardiogram demonstrated signs of right ventricular strain until the fourth post-operative day. Collateral veins were observed on the anterior abdominal wall two weeks after operation. Oedema did not develop in the lower limbs. The patient is now back at work and is able to undertake active exercise without distress.

CASE 2 A 43-year-old woman underwent a hysterectomy for menorrhagia so severe that her haemoglobin level on admission was $2.8 \mathrm{~g} . / 100 \mathrm{ml}$. A large dose of $\varepsilon$-aminocaproic acid had failed to control the haemorrhage. On the third post-operative day she suddenly became severely dyspnoeic with peripheral cyanosis and a systolic pressure of $100 \mathrm{~mm}$. $\mathrm{Hg}$.

There was a palpable right ventricular impulse and an apical third heart sound. There were no signs of venous thrombosis in the calves. The central venous pressure was $12 \mathrm{~cm}$. $\mathrm{H}_{2} \mathrm{O}$. Radiographs of the chest revealed oligaemic lung fields with prominent hilar

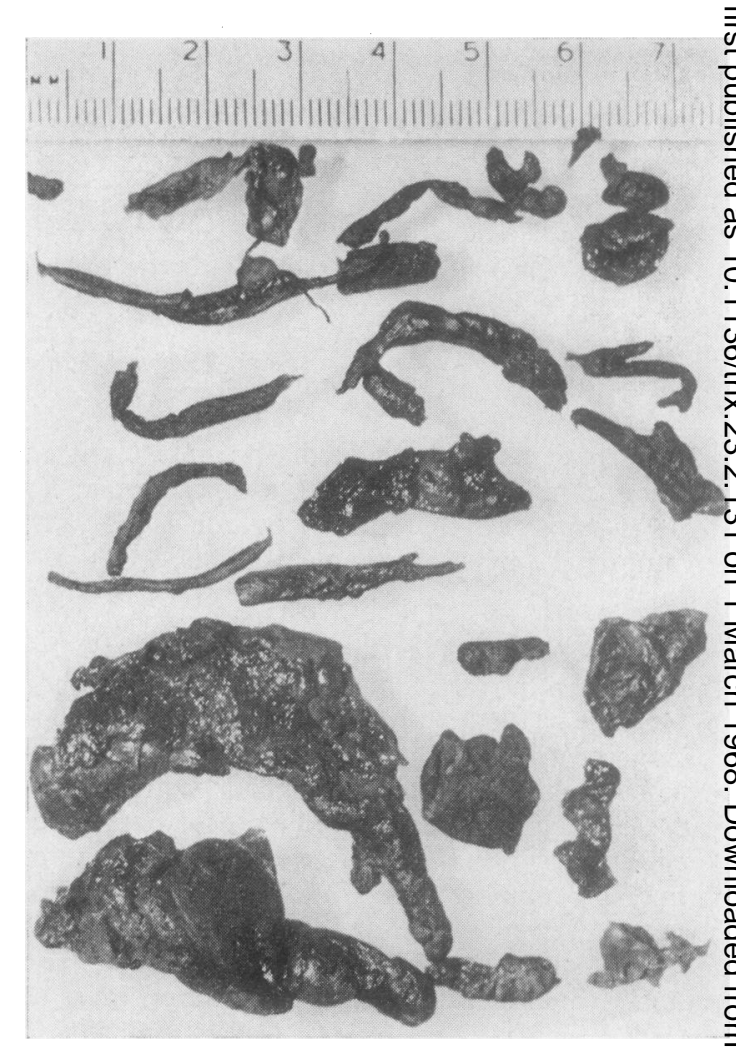

FIG. 1. Pulmonary emboli removed from case 1.

markings. The electrocardiogram showed ST depression in all leads. Pulmonary embolectomy was음 performed $1 \frac{1}{2}$ hours after the onset of symptoms, by $x$ which time the systolic pressure had fallen to $80 \%$ $\mathrm{mm}$. Hg. The pulmonary artery was explored during? a single period of venous inflow occlusion of 2 minutes 50 seconds at normothermia. Two emboli $16 \mathrm{~cm}$. long 3 and one $7 \mathrm{~cm}$. long, each with a diameter of $1 \mathrm{~cm}$., 0 were removed (Fig. 2). Immediately after the circula-5 tion had been restored the systemic pressure rose to $\frac{7}{2}$ $130 \mathrm{~mm}$. Hg. The inferior vena cava was narrowed. The electrocardiographic changes had virtually reverted to normal by the end of the operation and her post-operative course was uneventful. There was 0 some oedema of the left leg.

CASE 3 A man of 70 underwent a palliative resection of a carcinoma of the sigmoid colon with hepatic metastases. His progress was satisfactory until thes? eighth post-operative day, when he suddenly collapsed with signs of a massive pulmonary embolus. His con- $-\frac{0}{0}$ dition deteriorated and pulmonary embolectomy was carried out three hours after the onset of symptoms.? A large quantity of thrombus was removed from ${ }_{\odot}^{\mathbb{D}}$ the pulmonary artery using venous inflow occlusiono at normothermia. A marked improvement was 


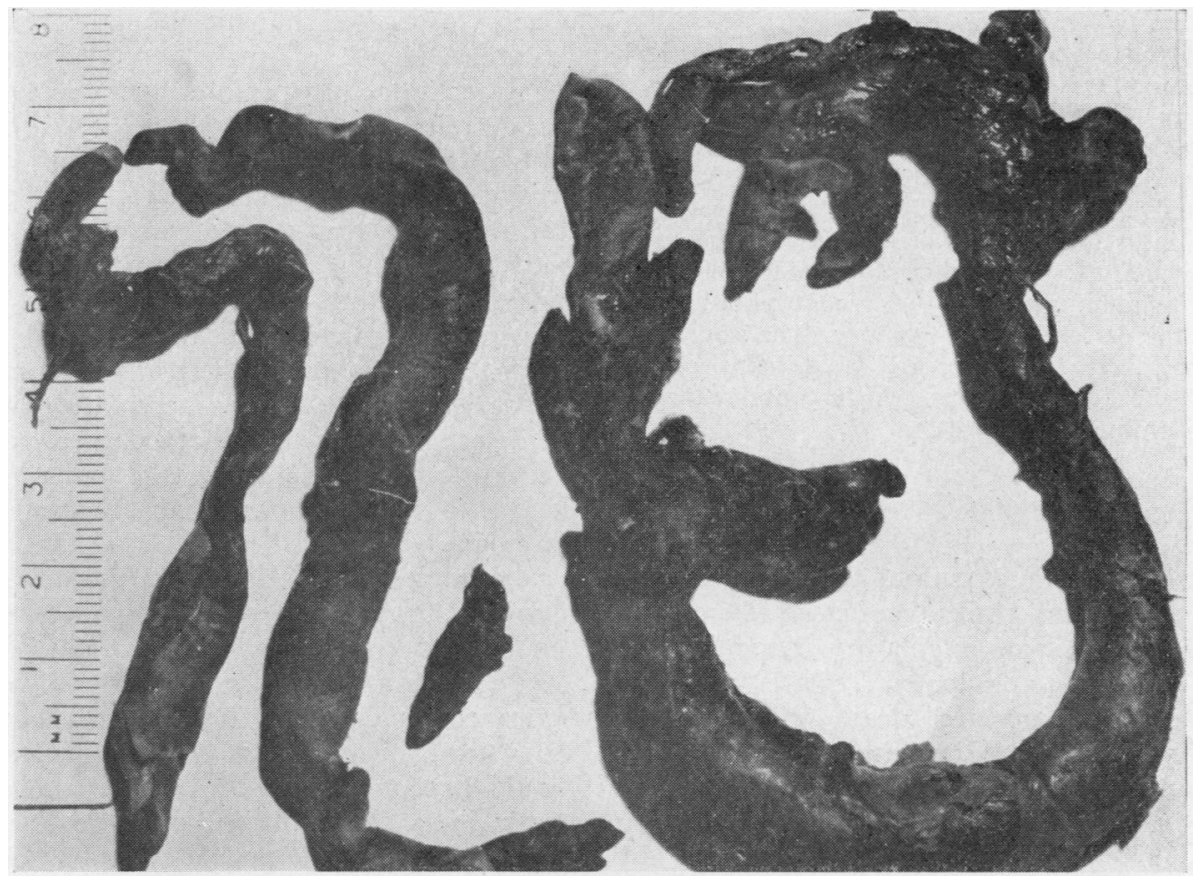

FIG. 2. Pulmonary emboli removed from case 2 .

observed following this, but two days later he developed bronchopneumonia. A tracheostomy was performed, but on the fifth day after embolectomy he became comatose with evidence of peripheral circulatory failure and died. At necropsy he was found to have a small perforation at the site of the rectosigmoid anastomosis with generalized faecal peritonitis. There was bilateral pulmonary venous thrombosis and residual emboli in the smaller branches of the pulmonary artery with pulmonary infarction.

CASE 4 A man of 47 was admitted for investigation of left renal colic. Two days later he suddenly felt faint while walking down the ward and rapidly became cyanosed with profound hypotension and tachypnoea. A clinical diagnosis of pulmonary embolism was made; further investigation was not undertaken because of swift deterioration, and he was operated upon within 30 minutes of the onset of symptoms. Cardiac arrest occurred while the sternum was being divided, and there was an interval of four minutes before effective cardiac massage could be started. The pulmonary artery was explored using venous inflow occlusion, and a $20 \mathrm{~cm}$. long pulmonary embolus was removed.

After restoring the circulation normal cardiac action was resumed and the systolic blood pressure on leaving the theatre was $120 \mathrm{~mm}$. $\mathrm{Hg}$. It soon became apparent that severe cerebral damage had been sustained. The patient was unconscious with spasticity of both lower limbs and generalized fits. Seven hours after operation the heart arrested and all resuscitative measures were unavailing. At necropsy a large quantity of clot was found in the pericardium and there was evidence of leakage from one end of the pulmonary artery suture line. The left pulmonary artery was free from clot but the right pulmonary artery was occluded by a coiled embolus. Roughening of the intima of the left external iliac vein indicated the origin of the emboli. The brain showed diffuse cerebral oedema with a prominent tentorial pressure cone.

CASE 5 A 54-year-old woman had undergone hysterectomy for carcinoma of the cervix. On the eighth post-operative day she developed the signs of pulmonary embolism sufficiently severe to produce inversion of $\mathrm{T}$ waves in leads II, III, aVF, and $\mathrm{V}_{4}$ to $V_{6}$. Treatment with anticoagulants was started. She was transferred to the Queen Elizabeth Hospital two days later and on arrival was found to be cyanosed and dyspnoeic with jugular venous distension, tachycardia, and a blood pressure of $110 / 60 \mathrm{~mm}$. Hg. The chest radiograph was equivocal. A sudden fall in blood pressure to $80 / 55 \mathrm{~mm}$. $\mathrm{Hg}$ next day, associated with evidence of increased right ventricular strain on the electrocardiogram, indicated that there had been a second episode of pulmonary embolism. The pulmonary artery was explored during 45 seconds of inflow occlusion, and two pieces of thrombus, each $10 \mathrm{~cm}$. in length, were removed. 
There were two brief periods of ventricular fibrillation, but effective ventricular contraction was restored and the blood pressure on return to the ward was $120 / 80 \mathrm{~mm}$. $\mathrm{Hg}$. The inferior vena cava was not ligated. She appeared to be making a satisfactory recovery until the sixth day after embolectomy, when she suddenly collapsed and died. At necropsy numerous infarcted areas were found in the right lower lobe and there was thrombus in the branches of the right pulmonary artery. A recent embolus was occluding the left pulmonary artery. The source of these emboli was found in the great veins of the pelvis and the femoral veins which contained antemortem thrombus.

\section{DISCUSSION}

Patients who sustain a pulmonary embolus may follow one of three clinical courses. There may be little disturbance of circulatory dynamics and recovery can be anticipated; there may be profound hypotension and death within a few minutes; or there may be severe depression of cardiac output and deterioration over the next few hours despite vasopressor agents and oxygen therapy. This latter group can be saved by pulmonary embolectomy.

Diagnosis of a massive pulmonary embolus is not always easy. Confusion between this and myocardial infarction is likely when the low cardiac output associated with embolism produces ischaemic changes on the electrocardiogram. Further, it may be difficult to distinguish between a single massive embolus and the profound effect of a small embolus on lungs which have been subjected to showers of small emboli over a long period.

The physical signs which are most useful in reaching a diagnosis are the severe dyspnoea, more severe than would be warranted by the minimal signs found on auscultating the lungs and inspecting the chest radiograph, and the presence of an apical third heart sound. The accentuated pulmonary second sound is an inconstant sign (Paneth, 1967). An elevated central venous pressure is confirmatory evidence. The chest film is seldom conclusive, but a paucity of pulmonary vascular markings may be noted. The electrocardiogram may reveal rhythm changes, prominent $\mathrm{P}$ waves, and the signs of right ventricular strain.

The accurate investigations of pulmonary embolism so well described by Sabiston and Wagner (1965) are unlikely to be available in the circumstances under which inflow occlusion pulmonary embolectomy would be carried out. Pulmonary angiography and scanning of the lung fields after an intravenous injection of ${ }^{131}$ I-tagged macro-aggregated human albumin provide con- $\overrightarrow{\vec{\omega}}$ clusive evidence of massive embolism, but the delay occasioned by performing these investigations on a rapidly deteriorating patient is only acceptable if some form of extracorporeal circulatory support can be provided. Serum enzyme studies and gas analyses to demonstrate a difference $\stackrel{\oplus}{0}$ between alveolar and systemic carbon dioxide $\overrightarrow{0}$ tensions are time-consuming. In this series the $\vec{\overrightarrow{ }}$ diagnosis was based on the physical signs, the $\vec{\omega}$ electrocardiogram, and the plain chest radiograph.

The decision to operate was made when signs $\times$ of deterioration were observed in a patient who $\underset{\omega}{N}$ had sustained a pulmonary embolus sufficiently ? $^{-}$ severe to produce profound changes in cardio- $\vec{\omega}$ vascular dynamics. It is conceded that the use of $\vec{o}$ cardiopulmonary bypass offers the best chance of $\frac{}{2}$ survival to a patient undergoing pulmonary embolectomy (Cooley, Beall, and Alexander, 1961 ; Paneth, 1967 ; Sautter, 1967), but in this $\stackrel{\widehat{S}}{3}$ series either deterioration was so acute that to $\vec{P}$ delay surgery until such measures were prepared $\mathscr{\mathscr { O }}$ would have been unacceptable or the emergency arose in a hospital without the facilities for providing extracorporeal circulation. The risks of performing pulmonary embolectomy must be balanced against a mortality of $50 \%$ in patients who are managed conservatively and an incidence $\stackrel{\mathbb{D}}{\triangle}$ of severe cardiac and pulmonary disablement in $\vec{\overrightarrow{ }}$ $30 \%$ of patients who survive such management. Just-Viera, Norwood, and Yeager (1967) pointed out the grave prognostic significance of hypotension and cyanosis after pulmonary embolus. They found that of 103 patients coming to necropsy $88 \%$ had been hypotensive and $64 \%$ cyanosed $\check{x}$

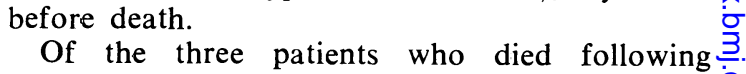
surgery, two were considerably improved by 8 removal of their emboli but succumbed as a result of faecal peritonitis in one and further pulmonary embolism in the other. The third patient died $\supset$ because cardiac arrest and irreversible cerebral 으․ damage occurred before obstruction of the pul- $\bar{N}$ monary artery could be relieved. Ligation of the 5 inferior vena cava might have saved one of these. $N$

Removal of emboli under inflow occlusion can $\underset{\omega}{N}$ never be as thorough or complete as under cardiopulmonary bypass, but sufficient thrombus 0 can be removed to revert the patient from an $\frac{\bar{\Phi}}{\Phi}$ expectation of certain death to an expectation of $\stackrel{\oplus}{+}$ probable recovery. Experimental studies indicate 0 that small pulmonary arterial radicles obstructed by residual thrombus can be expected to recanalize.

It is concluded that the performance of pul- $\frac{\varrho}{\sigma}$ monary embolectomy using a venous inflow 
occlusion technique at normothermia is an acceptable form of treatment in circumstances where cardiopulmonary bypass is not available.

I am grateful to Mr. L. D. Abrams for his permission to report cases 3, 4, and 5, and to Mr. T. Dee for the photography and reproduction of the electrocardiogram. I am also indebted to Mr. Selby Tait and Dr. Humphries, under whose care cases 1 and 2 were admitted.

\section{REFERENCES}

Allison, P. R., Dunnill, M. S., and Marshall, R. (1960). Pulmonary embolism. Thorax, 15, 273.

Cooley, D. A., Beall, A. C., and Alexander, J. K. (1961). Acute massive pulmonary embolism. Successful surgical treatment using temporary cardiopulmonary bypass. J. Amer. med. Ass., $177,283$.

Just-Viera, J. O., Norwood, T., and Yeager, G. H. (1967). Importance of shock and cyanosis in pulmonary embolism. Ann. Surg., $165,528$.

Kirschner, M. (1924). Ein durch die Trendelenburgsche Operation geheilter Fall von Embolie der Art. pulmonalis. Arch. klin. Chir., 133, 312.
Paneth, M. (1967). Pulmonary embolectomy. An analysis of 12 cases. J. thorac. cardiovasc. Surg., 53, 77.

Sabiston, D. C., and Wagner, H. N. (1965). The pathophysiology of pumonary embolism: relationships to accurate diagnosis and choice of therapy. Ibid., 50, 339.

Sautter, R. D. (1967). The technique of pulmonary embolectomy with the use of cardiop:almonary bypass. Ibid., 53, 268.

Sharp, E. H. (1962). Pulmonary embolectomy. Successful removal of a massive pulmonary embolus with the support of cardiopulmonary bypass-case report. Ann. Surg., 156, 1.

Steenburg, R. W., Warren, R., Wilson, R. E., and Rudolf, L. E. (1958) A new look at pulmonary embolectomy. Surg. Gynec. Obstet. $107,214$.

Trendelenburg, F. (1908). Ueber die operative Behandlung der Embolie der Lungenarterie. Arch. klin. Chir., 86, 686.

Vossschulte, K., Stiller, H., and Eisenreich, F. (1965). Emergency embolectomy by the transsternal approach in acute pulmonary embolism. Surgery, 58, 317.

\section{ADDENDUM}

A further two patients have undergone successful removal of pulmonary emboli with the use of normothermic venous inflow occlusion since this paper was submitted for publication. 